\title{
Applications of Calorimetric Techniques in the Formation of Protein-Polyelectrolytes Complexes
}

Diana Romanini, Mauricio Javier Braia and María Cecilia Porfiri

Additional information is available at the end of the chapter

http://dx.doi.org/10.5772/54260

\section{Introduction}

\subsection{Formation of the protein-polyelectrolyte complex}

Polyelectrolytes are flexible-chain polymers containing subunits with negative or positive charges. These compounds form soluble or insoluble complexes with proteins with opposite electrical charge.

The different equilibriums present in a solution of protein and polyelectrolyte are shown in figure 1.

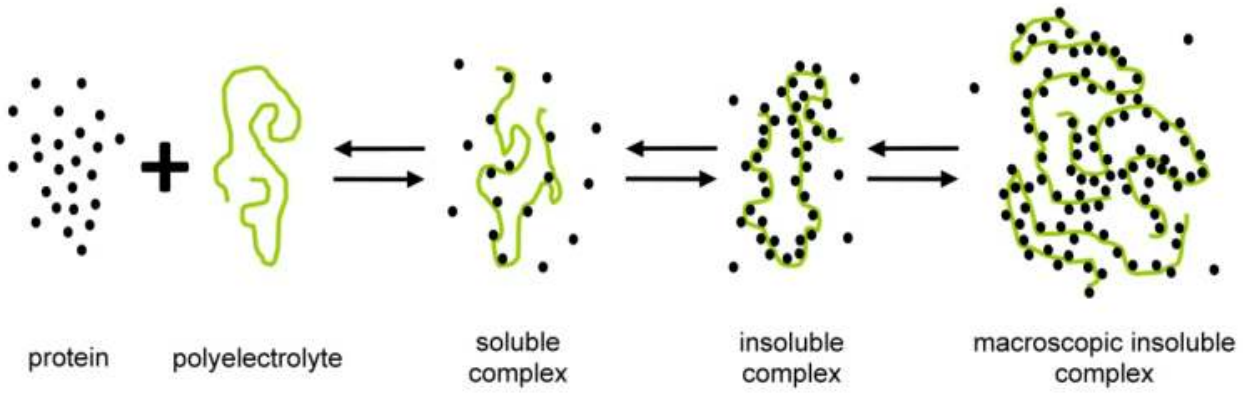

Figure 1. Formation of the protein- polyelectrolyte complex (binary complex).

As can be seen, when a protein interacts with a polyelectrolyte, a soluble complex is formed containing few molecules of the protein. As more molecules of protein interact with the polyelectrolyte, the complex becomes insoluble. Finally, the particles of insoluble complex start to interact with each other to form the macroscopic insoluble complex. 
Besides this model of formation of the insoluble complex, there is another model in which the formation of the insoluble complex requires the presence of an inorganic polyion (figure 2).

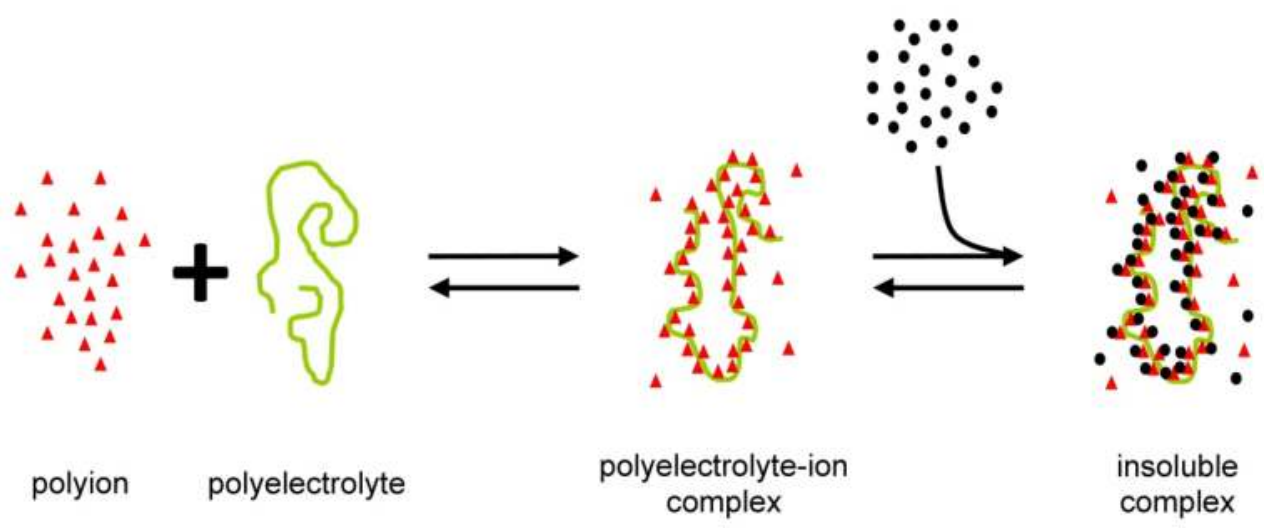

Figure 2. Formation of polyelectrolyte-ion-protein complex (ternary complex).

First, the polyelectrolyte interacts with the polyion and forms a soluble complex with charges oppose to those in the protein. Then, the protein interacts with the complex to form the insoluble complex.

In both cases, the formation and solubility of the complex depends on the $\mathrm{pH}$ and the ionic strength of the medium [1], the density of charges in the protein and the polyelectrolyte, the molecular weight and the concentration of the polyelectrolyte [2,3]. Various studies have been directed to understand the formation of these complexes in aqueous medium [4-6]. Equation 1 shows how the density of charges $(\sigma)$ on the surface of the protein and the polyelectrolyte is affected by the $\mathrm{pH}$ and the ionic strength of the medium.

$$
\sigma=\frac{\partial \sigma}{\partial \mathrm{pH}}(\mathrm{pH}-\mathrm{pI})
$$

Mattison et. al. postulated an equation that correlates the density of charges in the protein $(\sigma)$ and the polyelectrolyte $(\xi)$ with the Debye-Hückel constant $(\kappa)$ that is highly dependent on ionic strength ( $a$ is a constant that depends on the protein-polyelectrolyte system) [7].

$$
\xi \sigma \cong a \cdot \kappa
$$

The conditions of the medium determine whether the soluble or the insoluble complex is formed, or if the complex is dissociated.

\subsection{Stoichiometry of the protein-polyelectrolyte formation}

When studying the interaction of a protein and a polyelectrolyte, it is interesting to know the minimum quantity of protein requires forming the maximum quantity of complex per polyelectrolyte unit. This value is called stoichiometry of the complex $(\boldsymbol{e})$ and it is usually 
represented as the moles of polyelectrolyte per mol of protein, mass of polyelectrolyte per mass unit of protein, etc.

The stoichiometry of a protein-polyelectrolyte complex might be over or below 1 as shown in figure 3.

A complex with a stoichiometry below 1 contains more protein molecules than polyelectrolyte molecules. Usually, this kind of complex is insoluble, while a complex with a stoichiometry over 1 might be soluble or insoluble. As can be seen in figure 3, a complex with a stoichiometry below 1 can be turned into one with stoichiometry over 1 by adding polyelectrolyte to the medium. Of course, the effect can be reverted by adding protein.

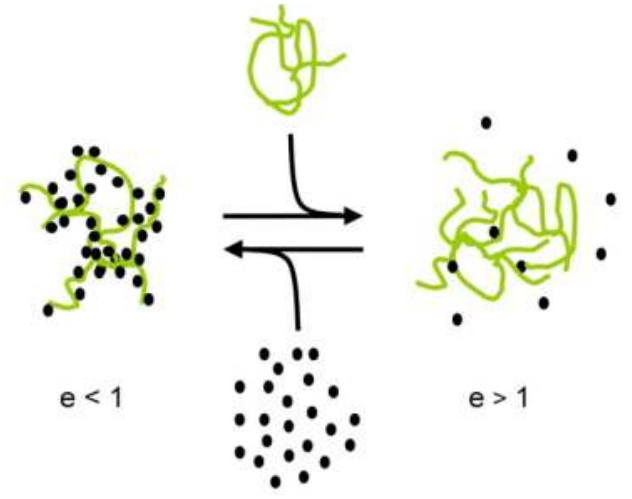

Figure 3. Stoichiometry $(e)$ of a protein-polyelectrolyte complex.

\subsection{Biotechnological applications of the protein-polyelectrolyte complex}

In biotechnology, it is interesting to use insoluble protein-polyelectrolyte complexes with e < 1 since they can be used to purificate and concentrate industrial-interest enzymes [8], to immobilized enzymes in bioreactors or scarefolds.

\subsubsection{Bioseparation of proteins from a complex mixture}

The development of Biotechnology has allowed the used of enzymes in the production of food, drugs and in many others industries. At the same time, Genetic Engineering and Molecular Biology has allowed the expression of proteins in bacteria and superior microorganisms; however, some proteins must still being isolated from its natural sources due to complex post-traductional modifications that occur during the synthesis of the proteins. In both cases, the protein of interest is in a media containing many other biomolecules and inorganic compounds that need to be separated from the protein before applied it to any industrial process.

Most purification protocols consist on many steps: the first ones have the aim to concentrate the protein of interest and to obtain a high recovery; while the last steps of the protocol are expected to yield a high purification factor. 
Precipitation of enzyme-polyelectrolyte insoluble complexes is a very useful technique to apply at the beginning of purification protocols since it requires simple equipment and so is very easy to scale up; needs low concentrations of the polyelectrolyte since it interacts with high affinity with the proteins; and can be based on a wide variety of polyelectrolytes, both natural and synthetic, positively or negatively charged. An important aspect of this technique is that the enzyme usually retains its tertiary structure and its catalytic activity. Even more, usually it is more stable in the presence of the polyelectrolyte $[9,10]$.

\subsubsection{Enzyme immobilization}

The immobilization of enzymes is a process by which the protein is attached to a solid matrix, synthesized using a polyelectrolyte, in order to enhance the stability of the structure of the protein and to reuse enzyme [11]. Enzymes immobilized, in comparison with enzymes in solution, are more robust and resistant to environmental changes.

Immobilization can be performed by physical or chemical methods. The first results in weak interactions between the enzyme and the solid support and includes adsorption on a waterinsoluble matrix and gel entrapment or micelles [12,13]. Chemical methods generate covalent bonds or electrostatic interactions between the enzyme and a water-insoluble support forming reticulated or single-chain particles. Insoluble complexes allow to immobilized enzymes by entrapment in polyelectrolyte solid particles, micelles or by covalent linkage to the support using carboimide as coupling.

The protein-matrix systems are widely used in bio-reactors for industrial process mainly because of the possibility of recycle the enzyme. Bio-reactors are very useful for the synthesis of organic compounds; since immobilized enzymes reduced the steps of the process, enhance the purity of the final products and allow stereo-selective synthesis. These systems also can be applied on the production of micro/nanocapsules for the delivery of proteins (or drugs). In this case, the use of polyelectrolytes sensitive to environmental conditions allows the releasing of the enzyme (or drug) molecules in different parts of the body [14].

\subsection{Characterization of the protein-polyelectrolyte complex}

The formation of the protein-polyelectrolyte complex can be studied by spectroscopic and calorimetric techniques.

Spectroscopy assays based on turbidimetric measurements allow knowing the effect of $\mathrm{pH}$, ionic strength, time and temperature on the amount of insoluble complex formed. Phase diagrams, turbidimetric titrations and kinetic assays must be performed in order to evaluate the best conditions to obtain the maximum quantity of insoluble protein-polyelectrolyte complex $[15 ; 16]$.

Study the stability of the enzyme when it is part of the complex is also important to understand how the polyelectrolyte affects its catalytic activity since it is expected to use the 
enzyme in an industrial process. Fluorescence, UV-visible and circular dichroism spectroscopy are very useful techniques to analyze the structure of the protein but also must be performed experiments to study the enzymatic activity.

Although these techniques are helpful, they do not give an idea of the nature of the interaction between the protein and the polyelectrolyte. Calorimetric techniques such as differential scanning calorimetry and isothermal titration calorimetry allow studying the thermal stability of the enzyme in the presence of the polyelectrolyte and the nature of the interaction between the enzyme and polyelectrolyte, respectively.

\section{Research course and methodology}

In order to study the formation of insoluble complexes between proteins and polyelectrolytes it is necessary to carry out different methodologies in a sequential way.

\subsection{Titration curves at different $\mathrm{pH}$ in binary systems}

The formation of the insoluble polyelectrolyte-protein complexes can be followed by means of turbidimetric titration of the protein with the polyelectrolyte, or vice versa. Taking into account the isoelectric point of the protein and the $\mathrm{pK}$ value of the polyelectrolyte, the $\mathrm{pH}$ of the medium should be selected so that they have opposite net charge.

Figure 4. shows the turbidimetric titration curve obtained for two systems with different behavior: hyperbolic and sigmoid. Turbidity is usually measured as the absorbance (Abs) at $420 \mathrm{~nm}$.
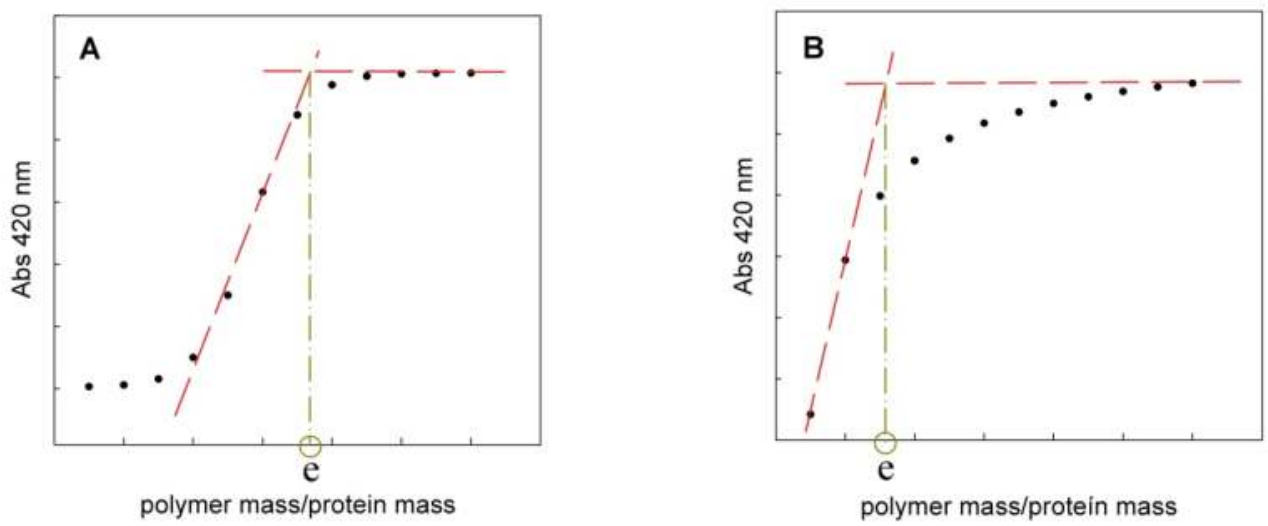

Figure 4. Determination of the polyelectrolyter/protein mass ratio when saturation is reached in a Asigmoid and B- hyperbolic graph

These graphs demonstrate a saturation behavior with different mechanism of complex formation, and allow us to determine the stoichiometric polyelectrolyte/protein ratio " $e$ ", which 
is defined as the minimal ratio in which the protein precipitates as an insoluble complex. The value " $e$ " is calculated from the plot at the lowest concentration of polyelectrolyte necessary to get the saturation. This value is important because it allows us to determine the minimal amount of polymer needed to fully precipitate the protein, and can be expressed as the number of moles of protein bounded per mol of polyelectrolyte.

\subsection{Titration curves at different $\mathrm{pH}$ in ternary systems}

In ternary systems, the polyelectrolyte forms an insoluble complex with an anion, which associates proteins with opposite net charge. The mixture polyelectrolyte-anion behaves as a pseudo polyampholyte with a characteristic isoelectric point.

The formation of insoluble complexes between the polymer and the anion can be examined by turbidimetric titration of the anion with the polyelectrolyte. When these curves reach the saturation it suggests a complete precipitation of the complex.

The precipitation of polyampholyte -protein complexes is driven by coulombic forces, which are highly dependent on protein and polyampholitic isoelectric $\mathrm{pH}$ values $[5 ; 17 ; 18]$. So, precipitation begins at a critical $\mathrm{pH}$ where the attractive forces have just overcome electrostatic repulsion.

\subsection{Phase diagrams in binary systems}

Phase diagrams, also called solubility curves, show the range of $\mathrm{pH}$ in which the complexes are soluble or insoluble. It means that they provide information about the $\mathrm{pH}$ of higher interaction between the components and the optimum $\mathrm{pH}$ for precipitation and dissolution of the complexes.

To obtain these diagrams, a polyelectrolyte/protein mixture at a ratio close to " $e$ " is titrated with acid or alkali and the turbidity of the medium is measured after $\mathrm{pH}$ variation.

Figure 5. shows an schematic phase diagram in a binary system.

\subsection{Phase diagrams in ternary systems}

Classical polyampholytes have both anionic and cationic groups in their molecules. However, the aqueous solution of any polyelectrolyte may behave as a polyampholyte provided there is a small ion with multiple electrical charges (two or more) in the medium which interacts with the opposite charge of the polyelectrolyte to form a pseudo polyampholyte. Under these conditions, it is possible to find a $\mathrm{pH}$ interval where the pseudo complex behaves as an ampholyte.

To obtain the phase diagrams, a mixture with fixed polyelectrolyte/anion ratio is titrated with alkali or acid, and the turbidity of the medium is measured as the absorbance at 420 $\mathrm{nm}$ after $\mathrm{pH}$ variation. 


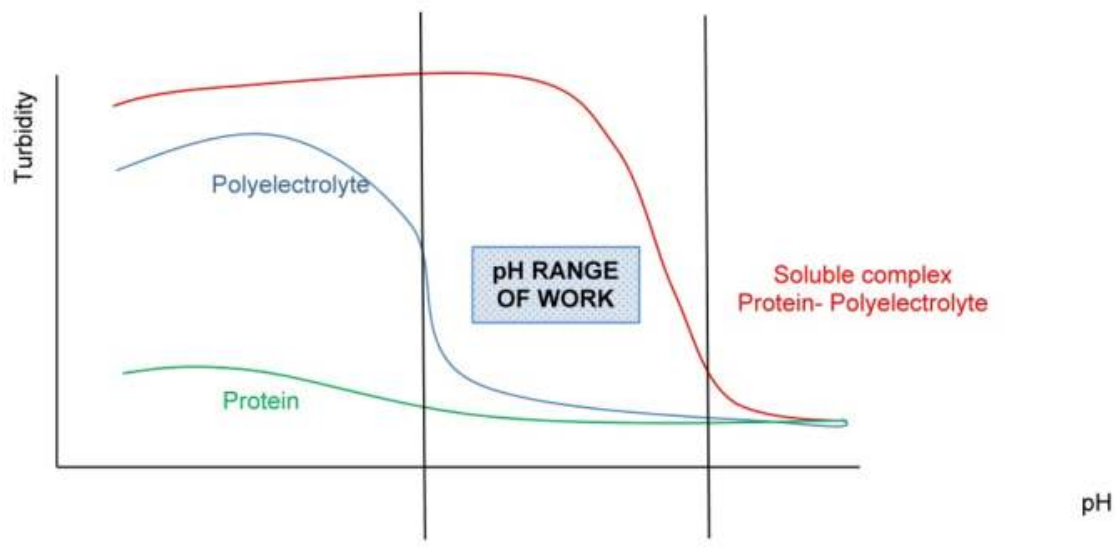

Figure 5. Phase diagram, turbidity vs. $\mathrm{pH}$, for protein $(-)$, polyelectrolyte $(-)$ and binary system $(-)$.

Figure 6 shows a schematic phase diagram between a cationic polyelectrolyte and an anion, in different polymer/anion ratios. Figure $6 \mathrm{~A}$ shows a $\mathrm{pH}$ range where the turbidity of the medium drastically increases. Each curve has a trapezoidal shape with a plateau, and the height of the trapezium depends on the polyelectrolyte concentration. The $\mathrm{pHs}$ that correspond to the edges of the trapezium are the critical $\mathrm{pH}$ values, at which the transition from complete dissolution to precipitation occurs. The lower critical $\mathrm{pH}$ is usually called acidic, and the higher one is called basic. It is remarkable that the transitions from complete solubility to precipitation occur at the same critical pHs independently of the polyelectrolyte concentration.

Also, phase diagrams can be represented as in figure 6.B. This diagram represents the behavior of the polyelectrolyte-anion complex by filled and open circles: filled circles are drawn at the $\mathrm{pH}$ of non-zero absorbance whereas the open circles at the zero absorbance values of the solution.

As mentioned above, insoluble polyelectrolyte-anion complexes behave as an ampholyte. This can be used to precipitate cationic or anionic proteins depending on the $\mathrm{pH}$ of work, as indicated in figure 6.B.

\subsection{Effect of ionic strength}

The coulombic component in the interaction between proteins and polyelectrolytes is closely related with the presence of charges. So, the ionic strength of the medium can alter the forces involved in the interaction and eventually leads to dissociation of the complexes.

In ternary systems, high ionic strength can also inhibit polymer-anion interaction.

Whatever the system, this inhibition of the formation of the precipitates may be directly proportional to salt concentration. So, the effect of the presence of salt in the systems can be evaluated by turbidimetric titration in the presence of different concentrations of $\mathrm{NaCl}$ in the medium. 

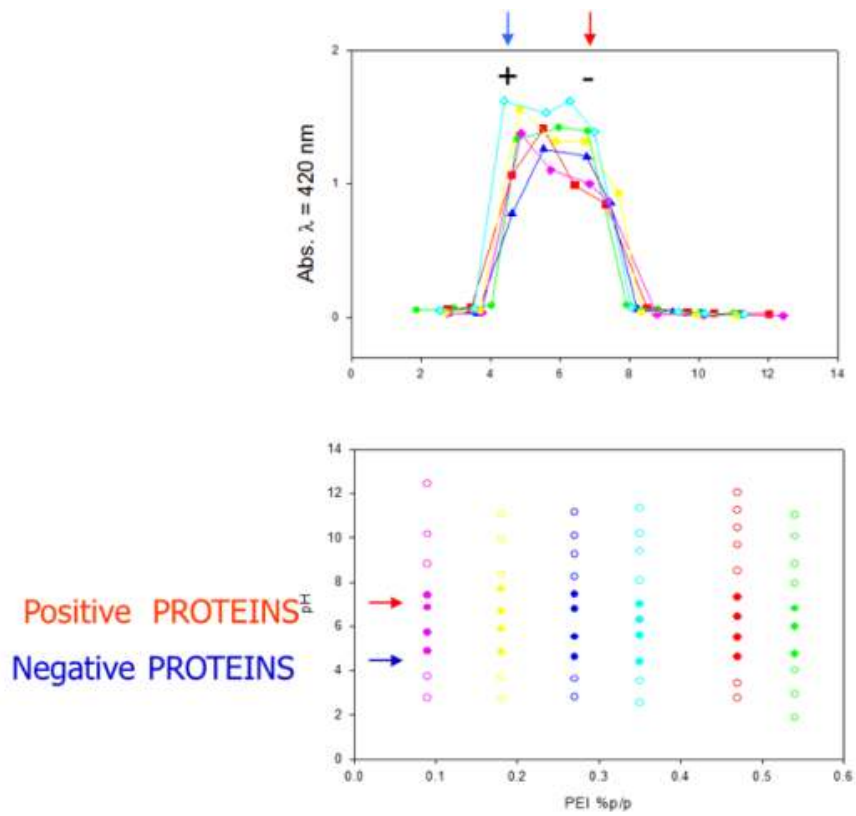

Figure 6. Phase diagram in polyelectrolyte-anion systems. Each color represents a different polyelectrolyte/anion ratio.

\subsection{Complex formation kinetics}

The interaction between polyelectrolytes and proteins requires time to achieve the maximum quantity of complex (maximum turbidity). Thus, it is necessary a kinetic study by which the turbidity of a mixture polyelectrolyte-protein is measured over time. So, a solution of the polyelectrolyte is added to a solution of the protein, at the $\mathrm{pH}$ of precipitation and in an appropriate ratio, and absorbance at $420 \mathrm{~nm}$ is measured over time. Finally, a plot of turbidity vs. time is made and the time required to reach the maximum quantity of turbidity is obtained [19].

\subsection{Conformational and enzymatic evaluation of the protein in the complex}

Several investigations have reported that polymers stabilize the catalytic activity in a variety of enzymes. Besides, it has been suggested that electrostatic interactions between the enzyme and polyelectrolytes play a primary role, also in conformational stabilization [20;21].

\subsubsection{Effect of the polyelectrolytes on the far-UV circular dichroism (CD) of proteins}

Circular dichroism spectroscopy is a very frequently used technique to evaluate protein conformation in solution. This method is sufficiently simple and allows a rapid determination of protein structure or conformational changes. 
In the far-UV region (between 180 and $250 \mathrm{~nm}$ ) the circular dichroism spectrum provides information one the secondary structure of proteins, due to asymmetrical packing of intrinsically achiral (planar) peptide groups [22].

The effect of polymers on the structure of proteins can be analyzed in terms of its secondary elements. So, far-UV circular dichroism spectra of proteins are recorded in different polymer/protein ratios, with a fixed concentration of protein and varying the amount of polymer. The $\mathrm{pH}$ of the medium must be the $\mathrm{pH}$ of higher interaction between the protein and the polyelectrolyte.

\subsubsection{Effect of the polyelectrolytes on the enzymatic activity of proteins}

In order to evaluate the effect of the polyelectrolyte on the enzymatic activity of the protein, enzyme assays are performed at constant protein concentration in the presence of different amounts of polymer. Polyelectrolyte/protein ratios are usually close to the stoichiometry of the complex (" $e$ ") or in excess of polymer respect this value.

The stability of the enzyme in the presence of the polyelectrolyte can also be monitored by incubating the mixture polyelectrolyte/enzyme and recording the enzymatic activity over time.

\subsection{Precipitation and redissolution of the complexes}

After analyzing the conditions of complex formation or dissolution and evaluating the effects of the different variables, we are able to design a methodology of precipitation of the protein with the polyelectrolyte by following the steps shown in figure 7.:

According to this precipitation scheme, an aliquot of the polyelectrolyte is mixed with a solution of the protein, both prepared at the $\mathrm{pH}$ of precipitation, to reach a proper polymer/protein ratio. This mixture is incubated the time necessary to form the maximum quantity of insoluble complex and centrifuged to obtain a solid precipitate. Then, the supernatant is separated and the precipitate redissolved in buffer at the $\mathrm{pH}$ of dissolution of the complex. Finally, in order to evaluate the effectiveness of the total process, enzymatic activity is measured in both fractions.

This scheme is successfully applied in many systems [15-20] and allows to obtain a protein with a high recovery and catalytically conserved.

\subsection{Calorimetric measurements for polymer-protein complex}

\subsubsection{Differential scanning calorimetry}

Thermal desnaturation of proteins was monitored with a high sensitivity differential scanning calorimeter model VP-DSC from MicroCal Inc. Thermograms were obtained between $20-85^{\circ} \mathrm{C}$, at scan rate $25-30^{\circ} \mathrm{C} / \mathrm{h}$ and at constant pressure of $28 \mathrm{psi}$. All result were averages of, at least, three independent measurements. Buffer versus buffer baseline scans were determined and subtracted from transition scans prior to normalization and analysis of 
protein denaturation. Finally, the values of the excess heat capacity were obtained after subtraction of the baseline. The calorimetric data were analysed by using the software ORIGIN 7.0, MicroCal Inc., following the methodology recommended by IUPAC. The parameters obtained from this analysis were: temperature at which maximum heat exchange occurs $(\mathrm{Tm})$, the area under the peak, which represents the enthalpy of transition for reversible process $\left(\Delta H_{c a l}\right)$ and the van't Hoff enthalpy $\left(\Delta H_{V H}\right)$.

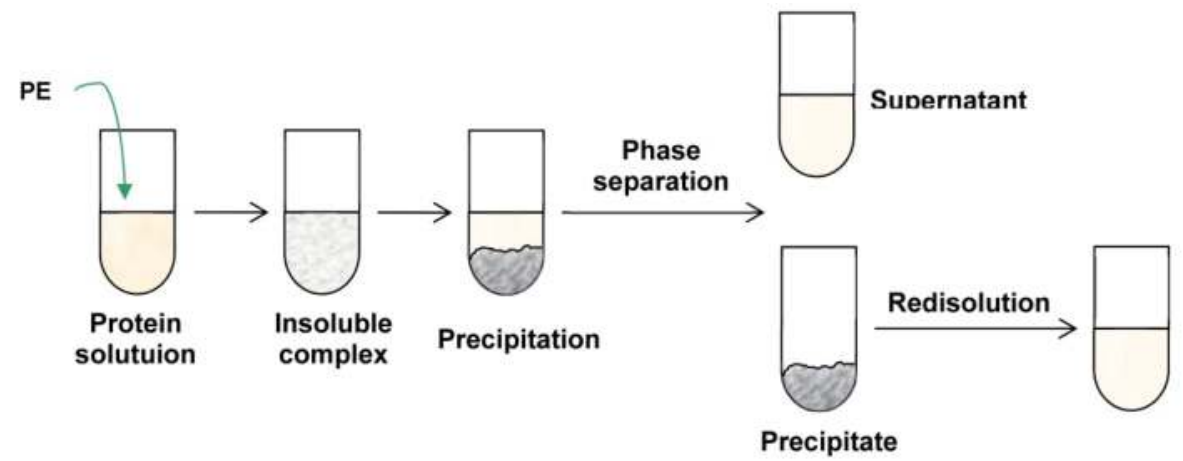

Figure 7. Scheme of precipitation of polyelectrolyte/protein complexes

The evaluation of $\Delta H_{v H}$ gives an idea of the mechanism of the unfolding process [23]:

- $\Delta \mathrm{HvH}_{\mathrm{v}}=\Delta \mathrm{H}_{\mathrm{cal}}$ : a two-state process is carried out under equilibrium condition.

- $\Delta \mathrm{HvH}_{\mathrm{v}}>\Delta \mathrm{H}_{\mathrm{cal}}$ : intermolecular cooperation is taking place which may require some degree of molecular association.

- $\Delta \mathrm{HvH}_{\mathrm{v}}<\Delta \mathrm{H}_{\text {cal: }}$ one or more intermediate states of significance in the overall process.

However, in some cases this calorimetric criterion may lead to erroneous conclusion.

\subsubsection{Isothermal titration calorimetry}

Measurements of the examples presented were performed at $20-25{ }^{\circ} \mathrm{C}$ by using a VP-ITC titration calorimeter (MicroCal Inc. USA). The sample cell was loaded with $1.436 \mathrm{~mL}$ of protein solution and the reference cell contained Milli-Q grade water. Titration was carried out using a $300 \mu \mathrm{L}$ syringe filled with polyelectrolyte solutions. The experiments were performed by adding aliquots of $3-5 \mu \mathrm{L}$ of polyelectrolyte solutions $0.175 \%(\mathrm{w} / \mathrm{w})$ to the cell containing the protein solution.

The mathematical model equation selected to fit the ITC data was derived from a model that assumes the polyelectrolyte molecule binding to several protein molecules, all with the same intensity; in other words, the polyelectrolyte was considered as a macromolecule having $\mathbf{n}$ independent and equivalent sites, all of which have the same affinity constant, $\mathrm{K}$, for the ligand (protein) [24].

The heat associated with the interaction polyelectrolyte-protein $\left(\Delta \mathrm{H}_{\mathrm{b}}\right)$ was calculated by subtraction using the equation 3 : 


$$
\Delta H_{b}=\Delta H_{t}-\Delta H_{d}-\Delta H_{\text {dissol }}
$$

Where $\Delta \mathrm{H}_{\mathrm{t}}$ is the total heat associated to each polymer addition, $\Delta \mathrm{H}_{\mathrm{d}}$ is the heat of dilution of the polyelectrolyte in the buffer in the absence of the protein and $\Delta \mathrm{H}_{\text {dissol }}$ is the heat of polymer dissolution. The heat associated to the dilution of the protein in buffer was negligible. Then $\Delta \mathrm{Hb}$ was plotted vs polyelectrolyte/protein molar ratio and, by non-linear fitting of these calorimetric curve, the affinity constant $(K)$ for the polyelectrolyte binding to the protein and the number of polymer molecules (n) bound per protein molecule was calculated using the software provided by the instrument.

The resulting data set was fitted using MicroCal ORIGIN 7.0 software supplied with the instrument and the intrinsic molar enthalpy change for the binding, $\Delta \mathrm{H}_{\mathrm{b}}$, the binding stoichiometry, $\mathrm{n}$, and the intrinsic binding constant, $\mathrm{K}$, were thus obtained. The equation for determining the heat associated to each injection is:

$$
Q=\frac{n M_{t} \Delta H_{b} V_{o}}{2}\left(1+\frac{1}{n k M_{t}}+\frac{X_{t}}{n M_{t}}-\sqrt{\left(1+\frac{1}{n k M_{t}}+\frac{X_{t}}{n M_{t}}\right)^{2}-\frac{4 X_{t}}{n M_{t}}}\right)
$$

where $V_{o}$ is the active volume cell, $\mathrm{X}_{\mathrm{t}}$ is the bulk concentration of ligand and $\mathrm{M}_{\mathrm{t}}$ is the bulk concentration of the macromolecule in $\mathrm{V}_{0}$ [25].

The intrinsic molar free energy change, $\Delta \mathrm{G}^{\mathrm{o}}$, and the intrinsic molar entropy change, $\Delta \mathrm{S}^{\mathrm{o}}$, for the binding reaction were calculated by the fundamental thermodynamic equations 5 and 6 :

$$
\begin{gathered}
\Delta G^{\circ}=-R T \ln K \\
\Delta S^{\circ}=\frac{\Delta H^{\circ}-\Delta G^{\circ}}{T}
\end{gathered}
$$

\section{Results}

\subsection{Turbidimetric titration curves}

Figure 8 shows typical hyperbolic titration curves of a protein with a polyelectrolyte. In this case, trypsin (TRP) with poly vinyl sulfonate (PVS) [26]. As can be seen, two important characteristics were observed: at low polymer/protein ratios, absorbance increases with an increase in the polyelectrolyte total concentration and, at high polyelectrolyte/protein ratio, there is a plateau which depends on the medium $\mathrm{pH}$.

The protein/polyelectrolyte molar ratio which corresponds to the situation where the protein has been precipitated as an insoluble complex was calculated from the intersection of a straight line which corresponds to the prolongation of the linear zone of the curve (at low polymer concentration) with a line which gives a plateau.

Trypsin is a serin-protease found in the digestive system. It is used for numerous biotechnological processes. Its isoelectric point is between 11.0 and 11.4 [27]. The pHs selected in the curves were chosen in the range where TRP and PVS have opposite charges. 


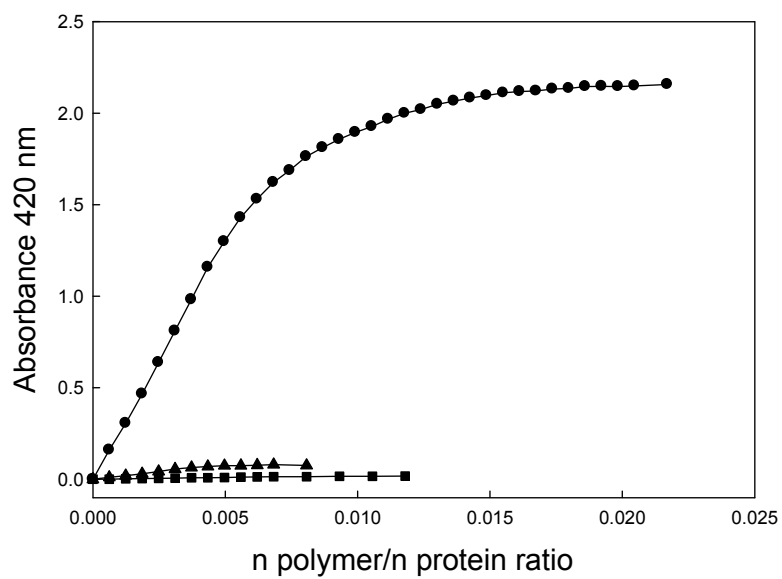

Figure 8. Turbidimetric titration curves of TRP $(70 \mu \mathrm{M})$ solution with PVS $(0.25 \% \mathrm{w} / \mathrm{w})$ in a medium with phosphate buffer $50 \mathrm{mM}$, pH $3.0(\bullet), 5.5(\boldsymbol{\Delta})$ and $7.0(\boldsymbol{\square}) . \mathrm{T}=20^{\circ} \mathrm{C}$, [21].

Table 1 shows the molar protein-polyelectrolyte ratio which corresponds to the stoichiometry of the complex formation calculated from the titration curves for the different experiments. These values are important because they allow us to calculate the minimal polymer amount necessary to precipitate the protein in a complete form. The data have been expressed as the number of TRP moles bound per polyelectrolyte mol. Despite the fact that these values were similar, turbidity is much higher at $\mathrm{pH} 3.00$ which suggest a major size of the precipitate particle.

\begin{tabular}{cc}
\hline $\mathbf{p H}$ & Protein/polyelectrolyte molar ratio \\
\hline 3.00 & $136 \pm 3$ \\
\hline 5.50 & $228 \pm 21$ \\
\hline 7.00 & $147 \pm 12$ \\
\hline
\end{tabular}

Table 1. TRP/PVS molar ratios at different $\mathrm{pHs}$.

Figure 9 shows titration curves of lysozyme (LYZ) with the polyelectrolyte PVS. LYZ is a basic protein with 19 amino residues, an isoelectrical $\mathrm{pH}$ between 11.0 and 11.4 and a molecular mass of $14.3 \mathrm{kDa}$ [28], therefore at the $\mathrm{pHs}$ where the turbidimetry titration were assayed the protein has a net positive electrical charge. Formation of LYZ-PVS complex was observed to be influenced by the medium $\mathrm{pH}$, however, at $\mathrm{pH} 3.1$, a minor absorbance maximum value was observed than at $\mathrm{pH} 5.5$, which can be assigned to the loss of the native structure of this protein by influence of the acid medium [26].

Table 2 shows the molar protein-polymer ratios which correspond to the stoichiometry of the complex formation calculate from the titration curves for the different experiments. These values are important because allow to estimate the minimal polyelectrolyte amount needed to precipitate the protein, the data have been expressed as the number of LYZ molecules bound per polyelectrolyte molecule. 


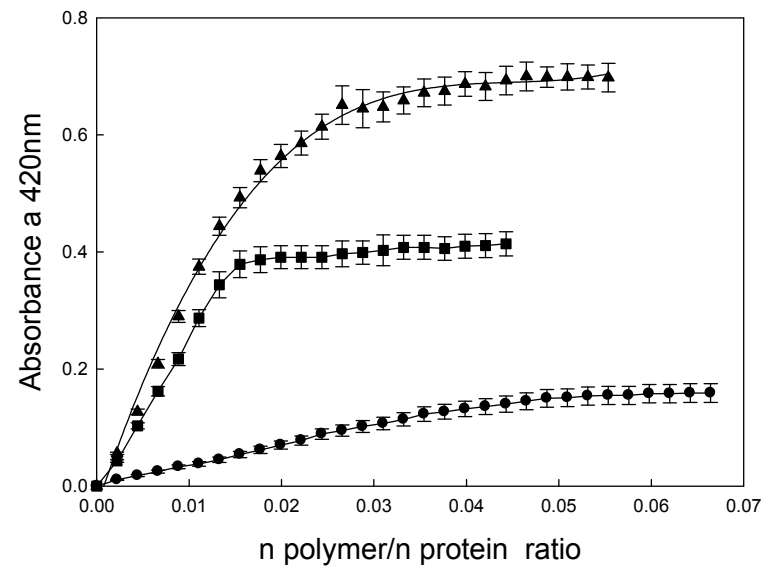

Figure 9. Turbidimetric titration curves of $\mathrm{LYZ}(0.3 \mathrm{mg} / \mathrm{mL})$ solution with PVS in a medium with $50 \mathrm{mM}$ phosphate buffer. pH $5.5(\boldsymbol{\Delta}), 7.0(\bullet)$, and acetic acid/acetate buffer pH $3.1(\boldsymbol{\square}) . \mathrm{T}=20{ }^{\circ} \mathrm{C}$.

\begin{tabular}{cc}
\hline LYZ- PVS & Protein/polyelectrolyte molar ratio \\
\hline pH 3.1 & 66 \\
\hline pH 5.5 & 47 \\
\hline pH 7.0 & 23 \\
\hline
\end{tabular}

Table 2. Lys/PVS molar ratios at different $\mathrm{pHs}$.

\subsection{Turbidimetric titration of ternary complex:}

Figure 10 shows turbidimetric titration curves when phosphate $(500 \mathrm{mM})$ or citrate $(50 \mathrm{mM})$ was titrated by adding a concentrated solution of the polyelectrolyte poly ethylene imine (PEI). Both curves reached a plateau at high polyelectrolyte anion ratios, which suggests a complete precipitation of the complex. It could be seen that the plateau was obtained at a polymer/anion ratio 10 times higher for citrate than for phosphate, suggesting that citrate has a greater precipitation capacity than phosphate. These ternary systems have the capability to precipitate in the absence of protein. Only is required the presence in the medium of the cationic polyelectrolyte (PEI) and a polyanion like phosphate (Pi) or citrate (Cit).

\subsection{Phase diagrams of binary systems}

Figures 11 show the absorbance dependence (at $420 \mathrm{~nm}$ ) vs. the $\mathrm{pH}$ change by the system LYS with poly acrylate (PAA). These complexes were soluble at basic $\mathrm{pH}$ values. At $\mathrm{pH}$ lower than 6.50 a significant increase in the turbidity was observed that corresponding to the insoluble complex formation. Similar behavior was reported for the serum albumin titration with anionic polyelectrolyte [7].

The relevance of these phase diagrams are in the possibility to know the insolubility and solubility complex conditions. 
$\mathrm{n} \mathrm{PEl/n}$ Cit ratio

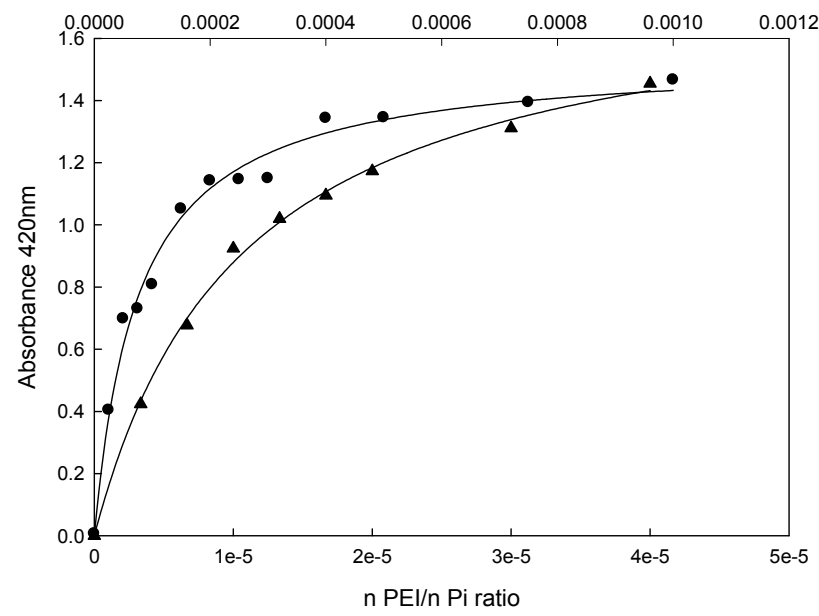

Figure 10. Turbidimetric titration of phosphate $(\mathbf{\Delta})$ and citrate $(\bullet)$ with PEI. pH 5.5. T=20ㅇ $\mathrm{C}[6]$.

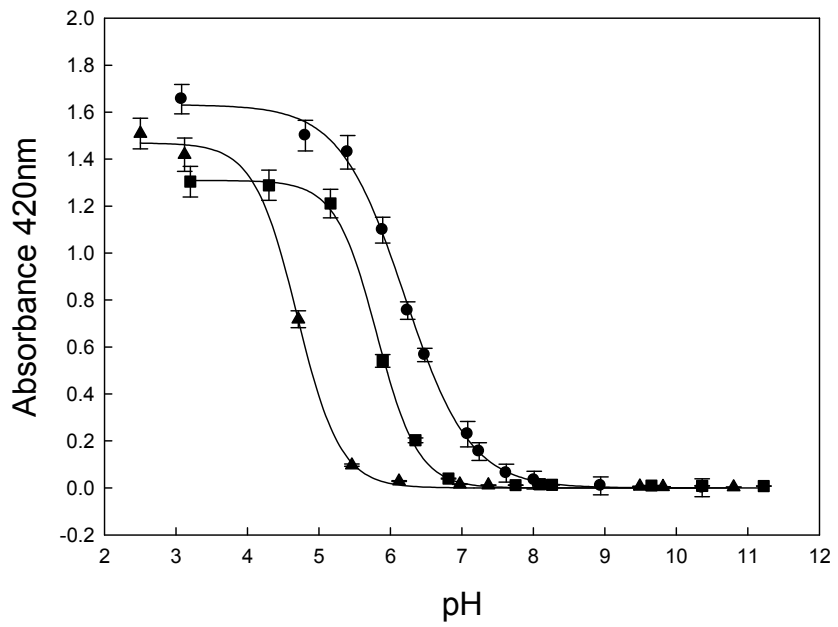

Figure 11. Dependence of the absorbance at $420 \mathrm{~nm}$ vs the medium $\mathrm{pH}$ at a constant proteinpolyelectrolyte molar ratio of LYZ-PAA: $(\bullet)$ 0.0027, $(\mathbf{\square}) 0.0065,(\boldsymbol{\Delta}) 0.0010$. T= 20ㄷ [25].

\subsection{Phase diagrams of ternary systems}

Figure 12 shows the $\mathrm{pH}$ variation effect on the insoluble complex formation obtained for ternary systems PEI/Pi at different PEI/Pi molar ratios [6]. As can be seen, in all curves there is an increase in the turbidity of the medium, reaching a maximum value and then decreasing in the $\mathrm{pH}$ interval 3.5-7. Each curve has a trapezoidal shape and the $\mathrm{pH}$ values corresponding to the edges of the trapezium are the critical pHs at which the transition from complete dissolution to precipitation occurs. 
In this figure it can be identify both critical pHs: 3.5 and 7 . At $\mathrm{pH}=3.5$ the net charge of the complex is positive whereas at $\mathrm{pH}=7$ is negative. On the other hand, transitions from complete solubility of the insoluble complex are independent of the polyelectrolyte concentration.

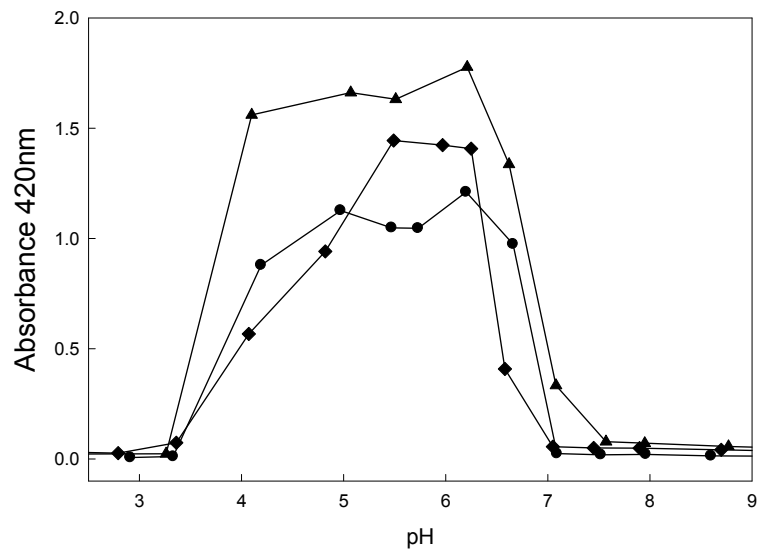

Figure 12. Phase diagram of PEI/Pi systems at different molar ratios.

\subsection{Effect of ionic strength}

In general, protein/polyelectrolyte insoluble complexes are greatly affected by ionic strength because the molecular mechanism of the interaction is mainly electrostatic in nature. Turbidimetric titrations at $\mathrm{pH} 7.00$ were performed in medium of different ionic strength such as shown Figure. 13. In this case, only $0.1 \mathrm{M}$ of $\mathrm{NaCl}$ is enough to avoid formation the insoluble protein-polyelectrolyte complex [26]. This finding may be interesting in the design of an isolation method of protein, allowing in a first step the precipitation by the polyelectrolyte and then the precipitate may be dissolved by $\mathrm{NaCl}$ solution addition at low concentration.

Ternary systems like PEI-citrate was dramatically affected by 0.5 for higher ionic strength; in this case, no formation of the insoluble complex was found while the PEI-phosphate system showed to be slightly affected by the $\mathrm{NaCl}$ increased concentration. The inhibition of the precipitate formation in both systems was directly proportional to the salt concentration, in agreement with the presence of an important coulombic component in the insoluble complex formation [19].

\subsection{Kinetics of the complex formation}

In general, the kinetics of complex formation is fast (around 2-10 minutes) [15;17;19]. Figure 14 shows the kinetic studies of different molar ratios of the systems TRP/Eudragit ${ }^{\circledR}$ L100 (EL100). It required less than 2 minutes of incubation to achieve the maximum quantity of complexes. In addition, by increasing the polyelectrolyte concentration increases the 
turbidity of the system, however the time required achieving the maximum turbidity is independent of the concentration of the molar ratio.

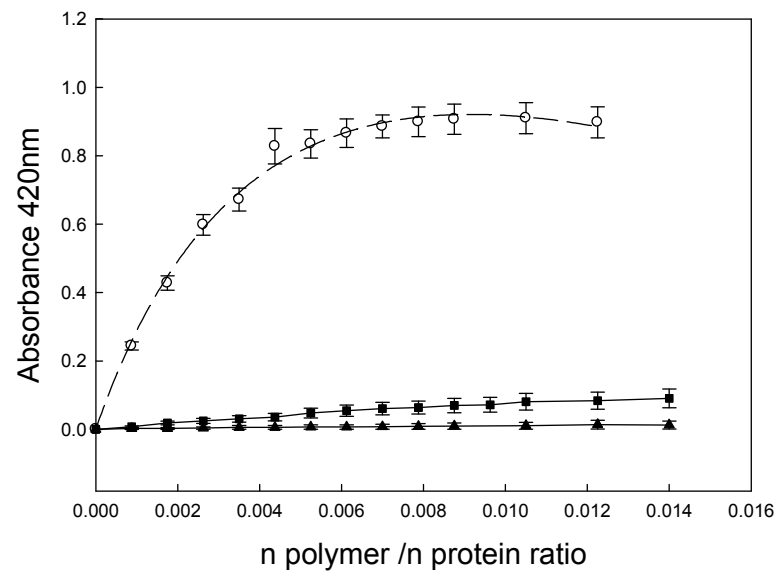

Figure 13. $\mathrm{NaCl}$ concentration effect on the turbidity of LYZ-PAA, $\mathrm{pH} 7.0, \mathrm{NaCl}$ concentration: $(\mathrm{O}) 0 \mathrm{M}$,

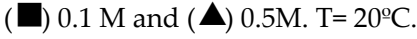

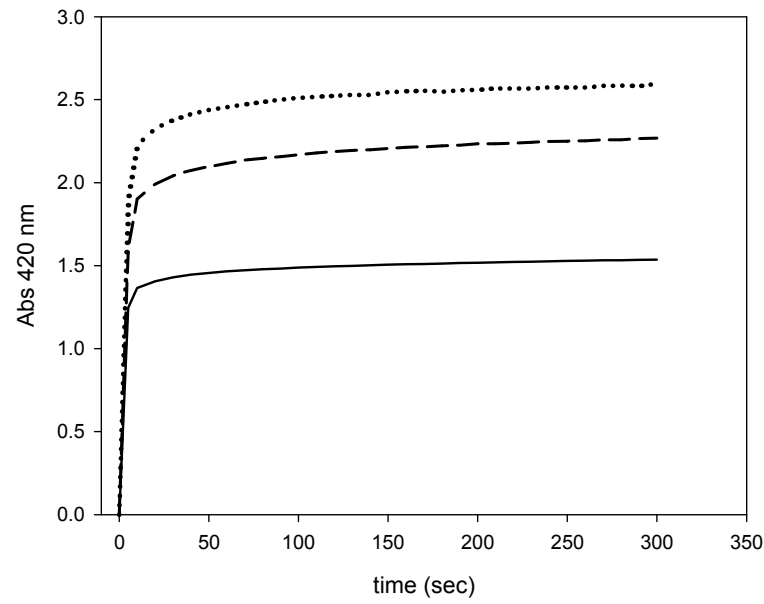

Figure 14. Formation of complex TRP-EL100 through time at three protein/polyelectrolyte molar ratio of TRP/EL100: $(-)$ 32.41, ( - - 16.18, (…) 8.08. [15].

\section{Calorimetric techniques of protein-polyelectrolyte complex}

\subsection{Differential scanning calorimetry by polymer-protein complex}

DSC is a useful tool for studying the protein unfolding in which values of excess specific heat capacity $(\mathrm{Cp})$ are obtained as a function of temperature. Two enzymes having different behavior towards charged flexible chain polyelectrolytes are analyzed below. 
Lysozyme is a basic protein with 19 amino residues, an isoelectrical $\mathrm{pH}$ between 11.0 to 11.4 and a molecular mass of $14.3 \mathrm{kDa}$. Because LYZ is one of the four proteins whose thermal denaturation is thermodynamically reversible, the equations for systems in thermodynamic equilibrium can be applied to obtain the thermodynamic functions (entropy and enthalpy of unfolding) directly from the thermograms, as described by Privalov [29].

Thermograms of LYS enzyme with PAA and PVS are presented as examples in figure 15 and Table 3 shows the thermodynamics functions and Tm obtained in each case. In these systems DSC measurements demonstrated that the Tm of LYS was not modified by the polyelectrolytes presence only a decrease in the denaturalization heat $\left(\Delta \mathrm{H}_{\mathrm{cal}}\right)$ was observed.

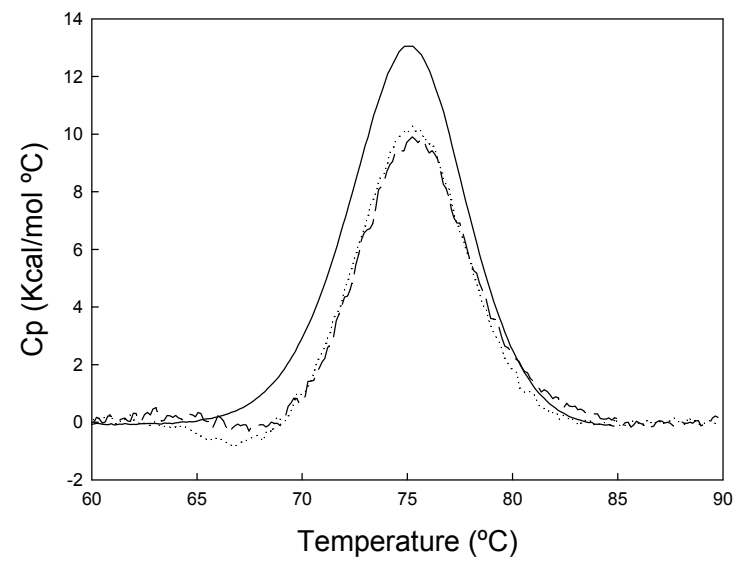

Figure 15. DSC Thermograms of the LYZ in the absence (-) and presence of: PVS (----) and PAA (....) pH 7.00 .

\begin{tabular}{|c|c|c|c|}
\hline & LYZ & LYZ-PVS & LYZ -PAA \\
\hline$\Delta \mathrm{Hcal}(\mathrm{kcal} / \mathrm{mol})$ & $89.4 \pm 0.3$ & $72.0 \pm 0.3$ & $66.7 \pm 0.4$ \\
\hline$\Delta \mathrm{HvH}_{\mathrm{H}}(\mathrm{kcal} / \mathrm{mol})$ & $139.0 \pm 0.6$ & $141 \pm 0.8$ & $151 \pm 1.0$ \\
\hline$\Delta \mathbf{H}_{\mathrm{vH}} / \Delta \mathrm{H}_{\mathrm{cal}}$ & $1.55 \pm 0.05$ & $1.96 \pm 0.01$ & $2.26 \pm 0.03$ \\
\hline $\operatorname{Tm}\left({ }^{\circ} \mathrm{C}\right)$ & $75.01 \pm 0.01$ & $75.33 \pm 0.02$ & $75.2 \pm 0.1$ \\
\hline$\Delta S$ (e.u.) & $399 \pm 3$ & $405 \pm 4$ & $405 \pm 5$ \\
\hline
\end{tabular}

Table 3. Thermodynamic functions obtained for the thermal LYZ unfolding determined by DSC in the absence and presence of the studied polyelectrolytes.

A Tm constant value is a proof that the protein retains its thermodynamic stability in the presence of both polyelectrolytes. However the polymer presence induced a decrease in the area under the curves, in agreement with a diminution of the heat associated to the denaturation process. The unfolding entropic change showed to be not affected by the polyelectrolyte presence, in accordance with the protein retaining its tertiary structure and no important conformational protein change is occurring. 
LYZ is a protein which has only one domain with low molecular mass, its thermal unfolding have been describe as reversible, however the capacity of LYZ to associate in aqueous solution it is well known. $\Delta \mathrm{HvH}_{\mathrm{H}} \Delta \mathrm{H}_{\mathrm{cal}}$ ratio greater than 1 is an indication of the intermolecular cooperation presence during the thermal unfolding. The increase of this ratio in the polyelectrolytes presence, suggests more cooperative intermolecular process.

Furthermore, the unfolding entropy was not affected in the protein-polymer complexes (LYZ-PVS and LYS-PAA). It indicates that LYZ in complex follows in the same conformational state that LYZ alone.

Trypsin is a serin-protease found in the digestive system. It is used for numerous biotechnological processes. It is a globular protein which has two domains with similar structures [27]. DSC experimental results for enzyme trypsin are demonstrated a two-state transition model at $\mathrm{pH} 3.00$ [30]. Figure 16.A shows DSC thermograms of TRP. Although the ratio $\Delta \mathrm{HvH}_{\mathrm{H}} \Delta \mathrm{H}_{\text {cal }}$ is close to 1 , however the thermogram clearly shows 3 transitions.

TRP-EL100 complex has a very interesting behavior. As can be seen in Figure 16.B protein thermogram was significantly modified by the polyelectrolyte presence.
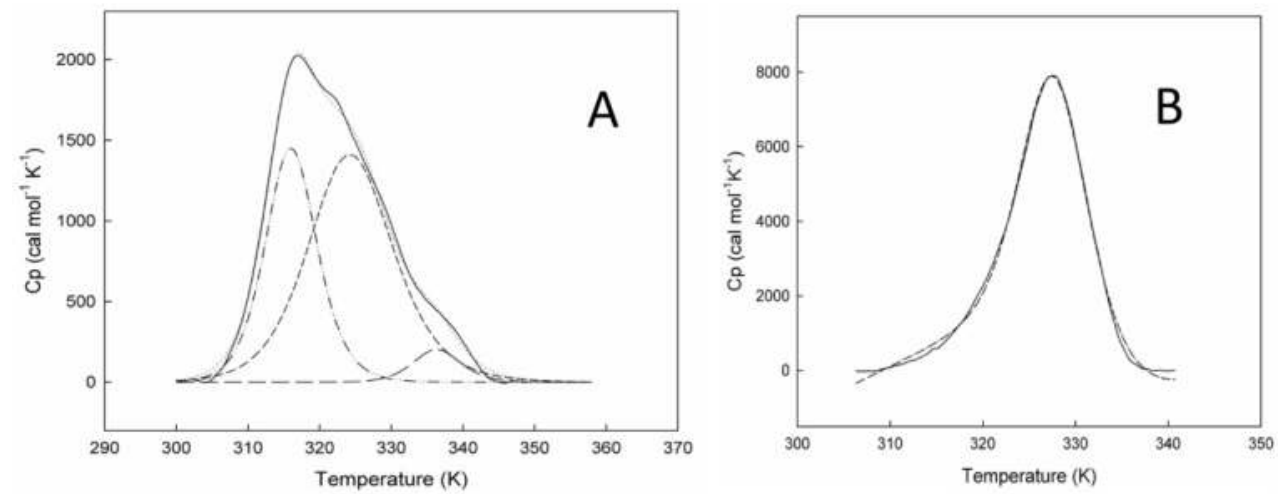

Figure 16. (A) DSC Thermogram of the TRP: (-) experimental data; (---) fit data; (..-) first transition; (- - ) second transition; (- - ) third transition. (B) DSC Thermogram of the TRP in the presence of EL100: (-) experimental data; (---) fit data.

\begin{tabular}{ccccc}
\hline & $\begin{array}{c}\mathbf{T m} \\
\mathbf{( K )}\end{array}$ & $\begin{array}{c}\Delta \mathbf{H}_{\mathrm{cal}} \\
(\mathbf{k c a l} / \mathbf{m o l})\end{array}$ & $\begin{array}{c}\Delta \mathbf{H v H}_{\mathbf{H}} \\
(\mathbf{k c a l} / \mathbf{m o l})\end{array}$ & $\Delta \mathbf{H v H} / \Delta \mathbf{H}_{\mathrm{cal}}$ \\
\hline TRP & $320.6 \pm 0.1$ & $38.7 \pm 0.6$ & $42.2 \pm 0.8$ & 1.09 \\
\hline \multirow{2}{*}{ TRP (transitions) } & $316.1 \pm 0.1$ & $13.9 \pm 0.1$ & $8.3 \pm 3$ & \\
& $324.4 \pm 0.3$ & $22.2 \pm 0.2$ & $53.1 \pm 3$ & - \\
\hline TRP-EL100 & $336.4 \pm 0.3$ & $1.8 \pm 0.5$ & - & 0.99 \\
\hline
\end{tabular}

Table 4. Thermodynamics functions obtained for the thermal TRP unfolding determined by DSC in the absence and presence of the EL100. 
The comparison of the two figures and table evidences two main differences

- $\quad$ Tm of the TRP curve is $320.6 \mathrm{~K}\left(47.5^{\circ} \mathrm{C}\right)$, whereas for the TRP-EL100 complex the same parameter is $327.8 \mathrm{~K}\left(54.6^{\circ} \mathrm{C}\right)$. The shift of the Tm of TRP in the presence of the polymer means that the EL100 stabilizes the structure of the enzyme against thermal denaturation.

- The different unfolding model in the absence (3 transitions of independent domains) and in the presence of polymer (two-state model)

\subsection{Isothermal titration calorimetry}

ITC technique gives the direct heat associated to the complex formation $(\Delta \mathbf{H})$, a number of protein molecules bounded to polyelectrolyte molecule (n), the affinity constant (K). Before

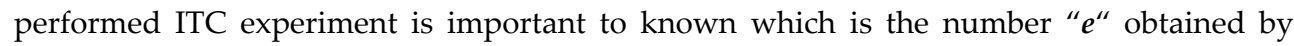
turbidimetric titration because is a good estimation of $\mathbf{n}$.

Figure 17 shows the ITC measurements of the LYS titration with PVS and Table 5 summarizes the parameters obtained by two anionic polyelectrolytes (PVS and PAA).
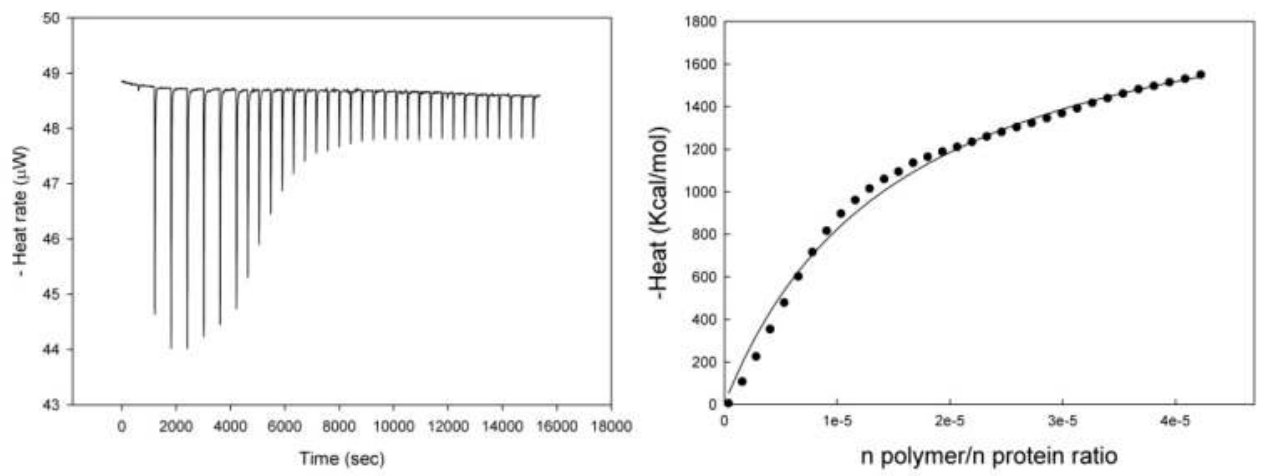

Figure 17. ITC measurements of LYZ with PVS [26].

\begin{tabular}{|c|c|c|}
\hline System & LYZ-PVS & LYZ -PAA \\
\hline n (protein /polyelectrolyte) & $21.2 \pm 0.2$ & $294 \pm 8$ \\
\hline$K\left(M^{-1}\right)$ & $2.710^{3}$ & $5.110^{4}$ \\
\hline$\Delta \mathrm{H}^{\mathrm{o}}(\mathrm{kcal} / \mathrm{mol})$ & -15.2 & -10.0 \\
\hline$\Delta S^{\circ}$ (e.u.) & -1103 & -1033 \\
\hline
\end{tabular}

*The enthalpic change is expressed per mol of protein bound.

Table 5. Thermodynamic and binding parameters of the interaction LYZ-polyelectrolyte from ITC experiments.

The interaction LYZ-PVS and LYZ-PAA is exothermic. The mechanism of bond is carried out between the electrically charged groups of both. The differences found between 
complexes were the affinity $(\mathrm{K})$ and the number of molecules of protein bonded to polymer molecule. Because the size of the polyelectrolytes are 10-fold larger than the protein, the number of protein molecules bound per polymer molecule is high.

The heat associated to the complex formation were extremely high, but when they are normalized per protein molecule bound to the polyelectrolyte the heat associate yielded 10$15 \mathrm{kcal} / \mathrm{mol}$ which is a normal heat amount for a coulombic interaction between two charge groups in solution. These low interaction heats are in agreement with the low $\mathrm{NaCl}$ concentration needed to induces the dissolution of the insoluble complex (around 0.1 M) Other important parameters to know are the thermodynamically stability of the protein in the polyelectrolyte presence. It is desirable that the protein retains its tertiary structure.

TRP- EL100 complex is an interesting example. Although the polymer and protein present opposite electrical charge, however the interaction is endothermic.

Figure 18 shows the binding isotherm obtained when consecutive aliquots of EL100 were added to a solution of trypsin [15]. The parameters calculated are summarized in Table 6.

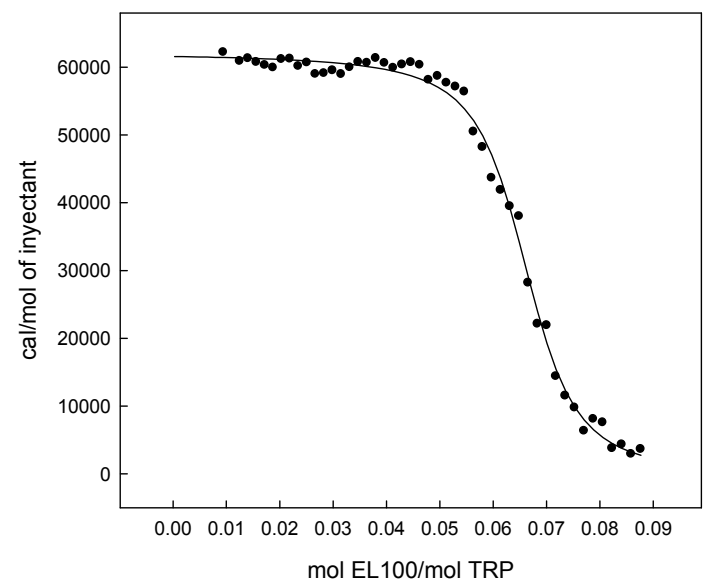

Figure 18. ITC measurements of TRP with EL100.

\begin{tabular}{lc}
\hline Binding parameter & Value \\
\hline$n$ ( molar ratio) $[\mathrm{mol} \mathrm{TRP} / \mathrm{mol} \mathrm{EL-100]}$ & $15.22 \pm 0.05$ \\
\hline$K$ (affinity constant) $\left[\mathrm{M}^{-1}\right]$ & $9.810^{6} \pm 7.10^{5}$ \\
\hline$\Delta H^{\circ}(\mathrm{kcal} / \mathrm{mol})$ & $62.1 \pm 0.3$ \\
\hline$\Delta S^{\circ}($ e.u. $)$ & $240 \pm 10$ \\
\hline$\Delta G^{\circ}(\mathrm{kcal} / \mathrm{mol})$ & $-9.59 \pm 0.05$ \\
\hline
\end{tabular}

Table 6. Binding parameters of the TRP- EL100 interaction from ITC experiments. T $=25^{\circ} \mathrm{C}$. 
A value of $15 \mathrm{~mol}$ of protein per mol of polyelectrolyte was found for the complex EL100TRP formation. The high value of the affinity constant demonstrated that both molecules interact strongly with each other. The $\Delta \mathrm{H}$ was normalized per mol of protein; therefore, heat value of $62.14 \mathrm{Kcal} / \mathrm{mol}$ of protein is yielded. The positive value of $\Delta \mathrm{H}$ indicates that the interaction between EL100 and TRP requires consuming of heat form de medium. The $\Delta S^{\circ}$ value obtained was positive as a result of the increase of the disorder of the system due to release of structured water molecules.

EL100 is a charged polymer which also contains a hydrophobic framework in its linear chain. For such a complicated system it is not clear to what extent non-electrostatic forces contribute to the observed complexation behavior. Besides, the value of $\Delta S^{\circ}$ was positive indicating that the disorder of the system increased.

ITC experiment performed in presence of $\mathrm{NaCl}$ confirmed the results obtained by turbidimetry (data not shown). The values of heat measured during the experiment of titration are similar that obtained when studying the dilution of the polyelectrolyte. This result is indicating that the TRP and the EL100 are not interacting when $\mathrm{NaCl} 1.00 \mathrm{M}$ is added to the buffer.

Thermodynamic parameters were according to hydrophobic interactions between TRP and EL100. However, ITC and turbidimetric titrations experiments were altered in salt presence. It would demonstrate that the mechanism of interaction between these two molecules involves both hydrophobic and electrostatic interactions.

\section{Conclusions}

Experimental conditions of charged polyelectrolyte-protein complex formation may be determined by turbidimetric measurements, but are necessary to complement it for calorimetric techniques.

DSC measurements show that the Tm and denaturalization heat of some proteins may increase or not change in the polymer presence and the complex unfolded according to a two-state model.

In general, $\Delta \mathrm{H}^{\circ}$ and $\Delta \mathrm{S}^{\circ}$ of complex formation obtained by ITC have negative when protein and polyelectrolyte are oppositely charged (electrostatic interaction). Nevertheless, the thermodynamic functions can be positive as a result of the interaction between hydrophobic backbone of polymers and aromatic amino acids. Moreover, if ionic strength modifies this insoluble complex formation, a mechanism of interaction may involve both hydrophobic and electrostatic interactions.

The calorimetric techniques (ITC and DSC), turbidimetry and enzymatic activity studies provide useful quantitative information about the interaction of proteins and charged polyelectrolytes in aqueous solution. The knowledge of the nature of this interaction is essential for the application of the complex formation in protocols as proteins isolation strategy, immobilization or in purification of a target protein. 


\section{Author details}

Diana Romanini, Mauricio Javier Braia and María Cecilia Porfiri

Laboratory of Physical Chemistry Applied to Bioseparation. College of Biochemical and

Pharmaceutical Sciences, National University of Rosario (UNR), Rosario, Argentina

\section{Acknowledgement}

We thank Prof Watson Loh, Institute of Chemistry, State University of Campinas (UNICAMP), Campinas, SP, BRAZIL for performing DSC and ITC measurements. We also thank Prof. G. Picó, Prof. B. Nerli and Prof. B. Farruggia for useful discussions.

\section{References}

[1] Kumara, A Srivastavaa A, Yu Galaevb I, Mattiasson B, (2007) Smart polymers: Physical forms and bioengineering applications. Progress in Polymer Science 32: 1205-1237.

[2] Wang, Y.; Gao, Y.; Dubin, P., (1999) Protein Separation via Polyelectrolyte Coacervation: Selectivity and Efficiency, Biotechnology Progress, 12: 356-362.

[3] Arvind, L.; Aruna, N.; Roshnnie, J.; Devika, T. (2000) Reversible precipitation of proteins on carboxymethyl cellulose, Poocess Biochemistry 35: 777-785.

[4] Weinbreck, F.; De Vries, R.; Schrooyen, P.; De Kruif, C.G. (2003) Complex Coacervation of Whey Proteins and Gum Arabic, Biomacromolecules, 4: 293-303.

[5] Gupta V., Nath S., Chand S., (2002) Estimation of proteins in the presence of polyethylenimine. Biotechnol. Lett., 22: 927.

[6] Manzur A, Spelzini D, Farruggia B, Romanini D, Picó G (2007) Polyethyleneimine phosphate and citrate systems act like pseudo polyampholytes as a starting method to isolate pepsin. Journal of Chromatography B. 860: 63-68.

[7] Mattison, K.W.; Dubin, P.L.; Brittain, I.J., (1998) Complex Formation between Bovine Serum Albumin and Strong Polielectrolytes: Effect of Polymer Charge Density, Journal of Physical Chemistry B, 102: 3830-3836.

[8] Cooper C, Dubin P , Kayitmazer A, Turksen S,Current, (2005) Polyelectrolyte-protein complexes Opinion in Colloid \& Interface Science 10: 52-78.

[9] Pessoa Jr., A.; Vahan Kilikian, B.;Purificação de Produtos Biotecnológicos, Ed. Manole Ltda., cap. 2. (2005).

[10] Hilbrig F, Freitag R, (2003) Protein purification by affinity precipitation, J Chrom B 790: 79-90.

[11] Arroyo M., (1998) Inmobilized enzymes: Theory, methods of study and applications. Ars Pharmaceutica, 39: 23-39.

[12] Krajewska B., (2004) Application of chitin- and chitosan-based materials for enzyme immobilizations: a review Enzyme Microb Tech 35: 126-139.

[13] Saskia Lindhoud. (2009) Polyelectrolyte Complex Micelles as Wrapping for enzymes Tesis, 206 p. 
[14] Esposito E., Cervellati F., Menegatti E., Nastruzzi C., Cortesi R., (2002) Spray dried Eudragit microparticles as encapsulation devices for vitamin C, Int J Pharm 242: 329334.

[15] Braia, M Tubio, G Nerli, B Loh W, Romanini, D., (2012) Analysis of the interactions between eudragit ${ }^{\circledR} 1100$ and porcine pancreatic trypsin by calorimetric techniques. Int J Biol Macromol 50: 180-186.

[16] Porfiri M. C., Picó G., Farruggia B., Romanini D., (2010) Insoluble complex formation between alpha-amylase from Aspergillus oryzae and polyacrylic acid of different molecular weight. Proc. Biochem. 45: 1753-1756.

[17] Tsuboi A., Izumi T., Hirata M., J. Xia, P. Dublin E. Kokufuta, (1999) Complexation of Proteins with a Strong Polyanion in an Aqueous Salt-free System Langmuir 12: 62956303.

[18] Fornasiero F., Ulrich J., Prausnitz J.,(1999) Molecular thermodynamics of precipitation. Chem. Eng. Process 38: 463-475.

[19] Patrickios, C, Sharma, L, Armes, S, Billingham, N. (1999) Precipitation of a WaterSoluble ABC Triblock Methacrylic Polyampholyte: Effects of Time, pH, Polymer Concentration, Salt Type and Concentration, and Presence of a Protein. Langmuir 15: 1613-1620.

[20] Foreman T, Khalil M, Meier P, Brainard J, Vanderberg L, Sauer N (2001). Effects of charged water-soluble polymers on the stability and activity of yeast alcohol dehydrogenase and subtilisin carlsberg. Biotechnol Bioeng 76: 241-246.

[21] Braia, M. , Porfiri, M.C., Farruggia, B., Picó G., Romanini, D. (2008) Complex formation between protein and poly vinyl sulfonate as a strategy of proteins isolation. Journal of Chromatography B, 873: 139-143.

[22] Fasman G. D. (1996) Circular dichroism and the conformational analysis of biomolecules. Plenum press 738p.

[23] Sturtevant J, (2001) Biochemical applications of differential scanning calorimetry. Annu. Rev. Phys. Chem. 38 463-488.

[24] Jha N, Kishore N., (2009) Binding of streptomycin with bovine serum albumin: Energetics and conformational aspects.Thermochim. Acta 482: 21-29.

[25] Kim W., Yamasaki Y., Kataoka K., (2006) Development of a Fitting Model Suitable for the Isothermal Titration Calorimetric Curve of DNA with Cationic Ligands. J. Phys. Chem. B 110: 10919-10925.

[26] Romanini D, Braia M, Giatte Angarten R, Loh W, Picó G, (2007) Interaction of lysozyme with negatively charged flexible chain polymers. J Chrom B, 857: 25-31.

[27] Beynon R, Bond J.S., Proteolytic Enzymes, Practical Approach, Oxford University Press, 2001.

[28] Aschaffenburg R., Blake C., Dickie H., Gayen, S., Keegan R., Sen A. (1980). The crystal structure of tortoise egg-white lysozyme at $6 \AA$ resolution .Biochim Biophys Acta 625: 64-71. 
128 Differential Scanning Calorimetry, Isothermal Titration Calorimetry and Microcalorimetry

[29] Privalov P.L..(1979) Stability of Proteins Small Globular Proteins. Adv. Protein Chem. 33: 167-241.

[30] Santos A. Santana M., Gomidea, F. Miranda A, Oliveira, J., Vilas Boas F., Vasconcelos, A., Bemquerer M. ,Santoro M., (2008) Physical-chemical characterization and stability study of $\alpha$-trypsin at $\mathrm{pH} 3.0$ by by differential scanning calorimetry Int. J. Biol.Macromol. 42: 278-284. 